\title{
LESSON 72
}

\section{Tabulation 28}

PAPER: A5 $(210 \times 148 \mathrm{~mm})$.

TARGET TIME: 20 minutes.

\section{Letter 34}

PAPER: A5 (148 × $210 \mathrm{~mm})$.

TARGET TIME: 15 minutes.

Take a carbon copy. Address a suitable envelope.

Display 6

PAPER: A5 $(148 \times 210 \mathrm{~mm})$.

TARGET TIME: 8 minutes.

Use small $x$ between the sections of this menu.

UNIT 18
OVEN TEMPERATURES

\begin{tabular}{|l|l|l|l|}
\hline Oven & Electricity F. & Gas Reguls & $\begin{array}{c}\text { Centigrade } \\
\text { Equivabent }\end{array}$ \\
\hline Cool & $225-250$ & $0-1$ & $107-121$ \\
Very slow & $250-270$ & $1-2$ & $121-135$ \\
Slow & $270-300$ & $2-3$ & $135-150$ \\
Vory Moderate & $300-350$ & $3-4$ & $150-175$ \\
Moderate & 375 & 5 & 190 \\
Moderntely Hot & 400 & 6 & 210 \\
Hot & $420-450$ & $7-9$ & $220-240$ \\
very Hot & $480-500$ & 9 & $250-260$ \\
\hline
\end{tabular}

Ref: DDIAR As for despatch to-day Gilbets Gregory, Esg., II Silver St. Otford,Dorset.D07 60T Dr Sr. Thnk $y$ f yr. enguiry cancening nateval stone poing slabs of walling blocks. (Paragraph) Full pooticulars are gun in $t$. endsd folder wh illustrater ou garden seupplies. We wd her to charge delivery on all ordes under $f 20.00 \mathrm{bec}$. of $t$ distance involued.

Yous faithfully Danid Denton Enc.

\section{MENU}

Oxtail Soup or Fruit Juice

Steak and Mixed Grill

Roast Duckling

Choice of Potatoes

Choice of Vegetables

Fruit and Cream or Mixed lces Coffee 